\title{
THE SURVIVAL RATE OF CHICK EMBRYO INFLUENCED BY NICOTINE AND ANTIOXIDANT GREEN TEA (CAMELLIA SINENSIS)
}

\author{
Maryam Shan, Shazia Muazam, Hina Shan*, Fahad Atta**, Zubia Iftikhar***, Amna Khalil**** \\ HBS Medical and Dental College Islamabad Pakistan, ${ }^{*}$ National University of Medical Sciences (NUMS) Rawalpindi Pakistan, ${ }^{*}$ Quaid Azam Medical College

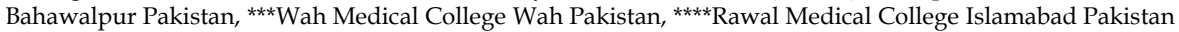

\begin{abstract}
Objectives: To study the effects of Nicotine on the survival rate of chick embryo and to evaluate the preventive role by the antioxidant green tea camellia sinensis.

Study Design: Laboratory-based experimental study.

Place and Duration of Study: Army Medical College, Rawalpindi, from Nov 2011 to Nov 2012.

Methodology: A total of 75 Fayoumi fresh fertilized eggs were kept at Poultry Research Institute, Rawalpindi. Experimental solutions were injected to the eggs of four groups at forty-eight hours of incubation and the effect on the growth was recorded and compared with the control group. The control and experimental groups were observed to assess the effects of Nicotine and the role of antioxidant green tea Camellia sinensis on the survival rate and growth of chick embryo's femur.

Results: The control group (group-1) showed 100\% survival rate of embryos. The experimental group-2 showed survival rate of $100 \%$ embryos and the group- 3 and group -4 showed survival rate $50 \%$ and $70 \%$ of embryos, respectively.

Conclusion: It was concluded that green tea decreases the oxidative stress caused by the Nicotine.
\end{abstract}

Keywords: Camellia sinensis, Chick embryo, Green tea, Incubation, Nicotine.

How to Cite This Article: Shan M, Muazam S, Shan S, Atta F, Iftikhar Z, Khalil A. The Survival Rate of Chick Embryo Influenced by Nicotine and Antioxidant Green Tea (Camellia Sinensis. Pak Armed Forces Med J 2021; 71(6): 2029-2032. Doi: https://doi.org/10.51253/pafmj.v6i6.6462

\footnotetext{
This is an Open Access article distributed under the terms of the Creative Commons Attribution License (https://creativecommons.org/licenses/by-nc/4.0/), which permits unrestricted use, distribution, and reproduction in any medium, provided the original work is properly cited.
}

\section{INTRODUCTION}

Every year 1.2 million deaths are attributable to this second-hand smoke exposure. Second-hand smoke is also related to devastating health conditions like cardiovascular diseases, respiratory diseases, low birth weight, and maternal depression. ${ }^{1}$ Exposure to secondhand smoke has been associated with chronic diseases such as chronic obstructive pulmonary disease and ischemic heart diseases. ${ }^{1,2}$ Evidence suggests that tobacco smoke exposure worsened allergic rhinitis in patients exposed to tobacco. ${ }^{2}$ People and children around the world and more importantly in low middle income countries are exposed to second hand smoke, especially at public places, hospitality venues, at home or in the vehicles.3,4 Many children are exposed by tobacco smoke both before and after birth making them vulnerable to the adverse health effects ranging from prenatal epigenetic changes, triggering diseases in childhood to adverse health effects in later life. ${ }^{4}$ Nicotine is an extremely addictive substance, and its safety or the potential of harming the health is not clear. Nicotine inhalation devices are gaining popularity among youth, highlighting the need for awareness and research on the cardiopulmonary risks of Nicotine and associated

Correspondence: Dr Maryam Shan, Department of Anatomy, HBS Medical and Dental College, Islamabad-Pakistan

Received: 17 Jul 2020; revision received: 09 Jul 2021; accepted: 12 Jul 2021 products exposure on health.5,6 Electronic cigarette (ecigarette) use has widely increased in recent years by non-smokers, including youth and young-adult nonsmokers.6 E-cigarettes are considered to be potentially effective as smoking cessation tool, however, dual use in smokers is common and e-cigarettes are widely used by non-smokers. Nicotine, the key addictive element in cigarettes, and also present in many e-liquids with varying quantities. Residue of tobacco smoke that lingers to indoor surfaces and clothing is known as third-hand smoke (THS) .6,7 Third-hand smoke has adverse effect on the growth and immunity of mice and has the potential to produce carcinogenic compounds. It has been reported that phylogenetic diversity of the home environment and alterations in the genera of the child microbiome are affected by active smoking and SHS and THS (e.g. staphylococcus, corynebacterium, streptococcus). 6,8

Research on the animals have shown the preventive roles for polyphenols in cardiovascular diseases, neurodegenerative diseases, cancer, diabetes and osteoporosis. ${ }^{9}$ Recent data supports the nutritional benefits of antioxidants in bone cells and/or in animals or selected groups of patients for the treatment and prevention of bone loss. Limited evidence shows dietary supplementation with green tea or Hypericum perforatum, containing various types of polyphenols, 
decreases trabecular bone loss and loss of collagen in bone matrix in ovariectomized rats. ${ }^{10}$ Animal research on the health effects of exposure to nicotine during pregnancy are needed to investigate the underlying relationship among oxidative stress, antioxidants, and bone metabolism. Our study investigates the effects of nicotine on the survival rate of chick embryo and evaluate the preventive role of the antioxidant green tea camellia sinensis.

\section{METHODOLOGY}

A laboratory based experimental study was carried out on 40 fertilized fyoumi eggs kept at Poultry Research Institute (PRI), Rawalpindi. The study was conducted at the Department of Anatomy, Army medical college Rawalpindi with a duration of one year, after IRB approval from Army medical college Rawalpindi. Fertilized fyoumi eggs were included at zero hour of incubation.

Inclusion Criteria: Fertilized eggs with normal shape (oval), colour (off-white) and size (medium) were included in the study.

Exclusion Criteria: Unknown time of laying, broken shell, abnormal shape (football shaped, pear shaped), colour (other than off-white) and size (very tiny eggs) were not included in the study.

The eggs were numbered and randomly divided through computer-generated technique into four groups. The four groups were labelled as 1, 2, 3, and 4 . Then through lottery method, groups were chosen as control and experimental groups. Insulin gauge needle was used to give working solutions. ${ }^{11}$ Group-1 was named as control group and was given normal saline; group-2 was given green tea extract; group-3 was given $0.0001 \%$ nicotine solution; and group-4 was given both $0.0001 \%$ Nicotine solution and green tea extract.

After complete procedure of fumigating and clearing the hatchery, incubation of eggs was started. The temperature was $37.5^{\circ} \mathrm{C}$ and $75 \%$ relative humidity maintained with proper ventilation. Rotations of eggs were done every 4 hours. Day zero was taken as the first time placement of eggs in hatchery. In the experimental groups, the solutions were administered by blunt shaped insulin gauge needle. The exposed embryos were compared with age matched control subgroups. After incubating the eggs for 17 days, ten eggs from each group were selected. The blunt end of eggs was opened with the help of forceps and embryos were collected. The embryos were separated from the yolk sac stored in the Formalin filled jars for48 hours. ${ }^{12,13}$

SPSS-22 was used for statistical analysis. Mean values and standard deviations were calculated for quantitative variables. One-way analysis of variance (ANOVA) was used to compare the four groups. The $p$-value of $\leq 0.05$ was considered statistically significant.

\section{RESULTS}

Total number of eggs were forty. The control group had no egg that does not reach the developing stage correctly it showed $100 \%$ survival rate of embryos. The experimental group-2 showed survival rate of $100 \%$ embryos and the group-3 and group-4 showed survival rate of embryos 5 out of 10 survived and 7 out of 10n survived in group-4 respectively (Figure).

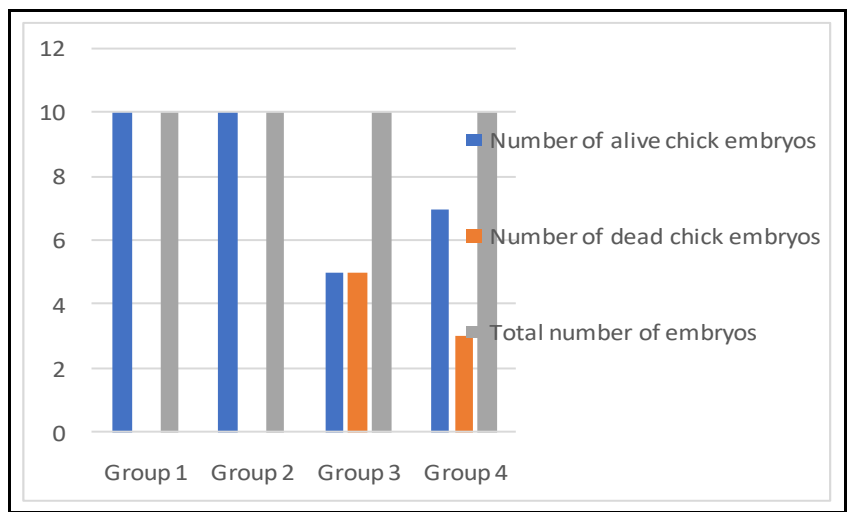

Figure: Number of chick embryo alive and dead among different groups of 17th day of incubation under the influence of nicotine and green tea. Goup-1 was given normal saline; Group-2 was given green tea extract; Group-3 was given $0.0001 \%$ nicotine solution; and Group-4 was given both $\mathbf{0 . 0 0 0 1 \%}$ Nicotine solution and green tea extract.

The comparison of control group-1 and experimental groups, group- 2 and group- 4 showed statistically insignificant results with $p$-value $(>0.05)$. The comparison of control group-1 and experimental group-3 showed statistically significant result with $p$-value (0.012). Experimental groups when compared with each other such as, group-2 in comparison with group3 and group- 4 showed $p$-value $(0.012)$ and $(0.063)$ respectively. Comparison of group-3 and group- 4 with each other showed statistically insignificant result with $p$-value (0.142) (Table-I \& II).

\section{DISCUSSION}

In our study once, the Nicotine was injected into the air sac of eggs after 24 hours of incubation, which resulted in embryos with low birth weight as well as smaller size and death of some embryos occurred due to paralysis and cardiac arrest. 
Table-I: Comparison of survival of chick embryo among different groups at 17th day of incubation.

\begin{tabular}{|c|c|c|c|c|c|c|c|}
\hline \multicolumn{2}{|l|}{ Parameters } & \multicolumn{2}{|c|}{ Group-1 $(n=10)$} & Group-2 (n=10) & Group-3 (n=10) & Group-4 $(n=10)$ & $p$-value \\
\hline \multicolumn{2}{|c|}{ Survival of Chick Embryo } & \multicolumn{2}{|c|}{$1.00 \pm 0.02$} & $1.00 \pm 0.1$ & $0.50 \pm 0.02$ & $0.70 \pm 0.1$ & $<0.001$ \\
\hline $\begin{array}{l}\text { Group } \\
\text { Comparisons }\end{array}$ & \multicolumn{2}{|c|}{$\begin{array}{c}\text { Group-1 } \\
\text { Vs. Group-2 }\end{array}$} & $\begin{array}{c}\text { Group-1 } \\
\text { Vs. Group-3 }\end{array}$ & $\begin{array}{c}\text { Group-1 } \\
\text { Vs. Group-4 }\end{array}$ & $\begin{array}{c}\text { Group-2 } \\
\text { Vs. Group-3 }\end{array}$ & $\begin{array}{c}\text { Group-2 } \\
\text { Vs. Group-4 }\end{array}$ & $\begin{array}{c}\text { Group-3 } \\
\text { Vs. Group-4 }\end{array}$ \\
\hline \multirow{2}{*}{$\begin{array}{l}\text { Survival of } \\
\text { Chick Embryo }\end{array}$} & \multicolumn{2}{|c|}{$p$-value } & $p$-value & $p$-value & $p$-value & $p$-value & $p$-value \\
\hline & \multicolumn{2}{|c|}{1.000} & 0.012 & 0.063 & 0.012 & 0.063 & 0.142 \\
\hline
\end{tabular}

Recent evidence suggests that Tobacco and Nicotine exposure lead to unique molecular and epigenomic (histologic and subcellular) placental modifications.11,14 Recently an investigation analyzed the mechanisms related to the retarded development of long bone and delayed endochondral ossification in rat fetus by prenatal nicotine exposure (PNE). ${ }^{15}$ Nicotine exposure is associated with adverse effects of gestational tobacco exposure, including diminished pulmonary function, auditory anomalies, impaired infant cardiorespiratory function, and cognitive/behavioral deficits in adult life. ${ }^{6-9}$ Oxidative stress changes bone remodelling course causing an imbalance between osteoblast and osteoclastactivity leading to metabolic bone diseases and pathogenesis of skeletal system disorders including osteoporosis characterized by low bone mineral density and decrease in bone mass. ${ }^{9}$ Recent evidence in a limited number of clinical studies have shown that antioxidant systems can be involved in the pathogenesis of bone loss. ${ }^{9}$ Polyphenols and anthocyanins are the antioxidants abundantly found in the diet especially in fruits, vegetables, cereals, dry legumes, chocolate, green tea, and coffee. 8,9

Similar results were reported from previous literature on the establishment of a chick embryo shellless culture system and its usage to observe changes in behaviour caused by Nicotine and substance from cigarette smoke. ${ }^{16}$ Increased intake of cigarettes has become a great health hazard for community with dangerous mental diseases. ${ }^{17}$ Cigarette smoking is a habit responsible of causing dangerous effects on all the system of human body. Individuals aged more than 60 and have a habit of cigarette smoking. They have two times more chances in mortality as compared with those who are nonsmoker. ${ }^{18}$ Quitting cigarette smoke as a first and secondhand smoker improved health benefits whereas some or all the reduced life expectancy can be improved depending on the age a person quits. ${ }^{14}$

Recent research by Kasote et al, showed the antioxidant property of some plants used as therapeutic entities. ${ }^{15}$ Green tea (Camellia sinensis) is the largely consumed tea in the world. Green tea extracts consumption provides polyphenols, which act as antioxidants. An antioxidant is a molecule capable of inhibiting the oxidation of other molecules. ${ }^{16}$ In another research work, animals which were treated with green tea, before giving to Nicotine, showed an improved response of nephroprotection, revealed by a significant decrease in oxidative damage. In our study, the threatening effect of Nicotine on the growth of chick embryo were reduced with Camellia sinensis however it can never overcome all the oxidative stress caused by the injection of Nicotine.

\section{CONCLUSION}

It was concluded that green tea decreases the oxidative stress caused by the Nicotine.

\section{Conflict of Interest: None.}

\section{Authors' Contribution}

MS: Main research work, SM: Literature review, HS: Refrences and data analysis, FA: Proof reading, ZI: Guiding and proof reading, AK: Proof reading.

\section{REFERENCES}

1. Sharma T, Khapre M. Exposure of secondhand smoke in women and children: A narrative review. J Family Med Prim Care 2021; 10(5): 1804.

2. Carreras G, Lugo A, Gallus S, Cortini B, Fernández E, López MJ, et al. Burden of disease attributable to second-hand smoke exposure: A systematic review. Prev Med 2019; 129(2): 105833.

3. Braun M, Klingelhöfer D, Oremek GM, Quarcoo D, Groneberg DA. Influence of second-hand smoke and prenatal tobacco smoke exposure on biomarkers, genetics and physiological processes in children-an overview in research insights of the last few years. Int J Environ Res Public Health 2020; 17(9): 3212.

4. Greene RM, Pisano MM. Developmental toxicity of e-cigarette aerosols. Birth Defects Res 2019; 111(17): 1294-1301.

5. Wetendorf M, Randall LT, Lemma MT, Hurr SH, Pawlak JB, Tarran R, et al. E-Cigarette exposure delays implantation and causes reduced weight gain in female offspring exposed in utero. J Endocr Soc 2019; 3(10): 1907-1916.

6. Suter MA, Aagaard KM. The impact of tobacco chemicals and nicotine on placental development. Prenat Diagn 2020; 40(9): 1193-200.

7. Hu H, Zhao X, Ma J, Shangguan Y, Pan Z, Chen L, et al. Prenatal nicotine exposure retards osteoclastogenesis and endochondral ossification in fetal long bones in rats. Toxicol Lett 2018; 295(1): 249-255.

8. England LJ, Aagaard K, Bloch M, Conway K, Cosgrove K, Grana $\mathrm{R}$, et al. Developmental toxicity of nicotine: A transdisciplinary 


\section{Antioxidant Green Tea}

synthesis and implications for emerging tobacco products. Neurosci Biobehav Rev 2017; 72(1): 176-189.

9. Domazetovic V, Marcucci G, Iantomasi T, Brandi ML, Vincenzini MT. Oxidative stress in bone remodeling: role of antioxidants. Clin Cases Miner Bone Metab 2017; 14(2): 209-216.

10. Shan M, Qamar K, Iqbal I. Role of nicotine and camellia sinensis on the developing femur of chick. J Pak Med Assoc 2015; 65(10): 1094-1096.

11. Hamamichi S, Nishigori H. Establishment of a chick embryo shell-less culture system and its use to observe change in behavior caused by nicotine and substances from cigarette smoke. Toxicol Lett 2001; 119(2): 95-102.

12. Schwartz AG, Cote ML. Epidemiology of Lung Cancer. Adv Exp Med Biol 2016; 893(1): 21-41.

13. Glasheen C, Hedden SL, Forman-Hoffman VL, Colpe LJ. Cigarette smoking behaviors among adults with serious mental illness in a nationally representative sample. Ann Epidemiol 2014; 24(10): 776-780.
14. Goldman DP, Zheng Y, Girosi F. The benefits of risk factor prevention in Americans aged 51 years and older. Am J Public Health 2009; 99(11): 2096-2101.

15. Kasote DM, Katyare SS, Hegde MV, Bae H. Significance of antioxidant potential of plants and its relevance to therapeutic applications. Int J Biol Sci 2015; 11(8): 982-991.

16. Vishnoi H. Green Tea (Camellia sinensis) and its antioxidant property: A review. Int J Pharm Sci Res 2018; 9(5): 1723-1736.

17. Begum MS, Saradamma B, Reddy VD, Padmavathi P, Maturu P, Ellutla $\mathrm{N}$, et al. Influence of green tea consumption on cigarette smoking-induced biochemical changes in plasma and blood. Clin Nutri 2017; 16(1): 1-2.

18. Songnuy T, Scholand SJ, Panprayoon S. Effects of Tobacco Smoke on Aeroallergen Sensitization and Clinical Severity among University Students and Staff with Allergic Rhinitis. J Environ Public Health. 2020, [Internet] Available at: https://www. hindawi.com/journals/jeph/2020/1692930/ (Accessed on January 10, 2020). 